\section{Atividade física em adultos e idosos residentes em áreas de abrangência de unidades básicas de saúde de municípios das regiões Sul e Nordeste do Brasil}

\author{
Physical activity in young adults and the elderly in \\ areas covered by primary health care units in \\ municipalities in the South and Northeast of Brazil
}

\author{
1 Universidade Federal de \\ Pelotas, Pelotas, Brasil. \\ 2 Universidade Católica de \\ Pelotas, Pelotas, Brasil. \\ 3 Secretaria Municipal da \\ Saúde, Pelotas, Brasil. \\ Correspondência \\ F. V. Siqueira \\ Programa de Pós-Graduação \\ em Epidemiologia, \\ Universidade Federal \\ de Pelotas. \\ Av. Duque de Caxias 250, \\ Pelotas, RS \\ 96030-002, Brasil. \\ fcvsiqueira@uol.com.br
}

\begin{abstract}
Shifts in Brazil's demographic structure have expanded the country's elderly population and consequently increased the rates of chronic diseases. This paper describes the prevalence of sedentary lifestyle and associated factors in a cross-sectional study, including 4,060 non-elderly and 4,003 elderly adults in 41 cities in seven States of the country. Prevalence of sedentary lifestyle was $31.8 \%$ (95\%CI: 30.4-33.2) in nonelderly adults and 58.0\% (95\%CI: 56.4-59.5) in the elderly; sedentary lifestyle was more frequent in the Northeast and among males. Low family income was associated with higher prevalence of sedentary lifestyle in both non-elderly and elderly adults, while low schooling was only observed among the elderly. There was an inverse association between self-reported health status and sedentary lifestyle. Mean prevalence of sedentary lifestyle was higher in areas covered by pre-PROESF units in comparison to other health system models. We conclude that sedentary lifestyle is highly prevalent and that disadvantaged socioeconomic groups are more likely to be sedentary.
\end{abstract}

Motor Activity; Heath Centers; Adult; Aged.
Fernando V. Siqueira 1,2

Luiz Augusto Facchini 1

Roberto X. Piccini 1

Elaine Tomasi 2,3

Elaine Thumé 1

Denise S. Silveira 1,3

Pedro C. Hallal 1

\section{Introdução}

O aumento da expectativa de vida da população e a diminuição das taxas de fertilidade têm determinado modificações na pirâmide demográfica brasileira. O envelhecimento populacional e o controle das doenças infecciosas têm gerado um aumento proporcional das doenças crônicas 1,2 Estima-se que até 2020 o número de idosos no mundo irá dobrar; no Brasil, projeta-se que, nessa época, $11 \%$ da população terá 60 anos ou mais (Instituto Brasileiro de Geografia e Estatística - IBGE. Censo Demográfico 2000. http://www. ibge.gov.br).

Com o aumento proporcional das doenças crônicas, existe a necessidade da preparação e adequação dos serviços básicos de saúde, visando a melhor estruturação, formação e qualificação profissional para o atendimento dessa nova demanda. Embora o sistema de saúde atual ofereça atendimento universal à população, problemas relacionados à escassez de recursos humanos, garantia de acesso, utilização de serviço, eqüidade no atendimento, têm sido descritos, tornando importante corrigi-los com o objetivo de melhor atender a população ${ }^{3}$.

Como uma opção importante na prevenção e tratamento de diversas doenças crônicas de alta prevalência em adultos e idosos, vários trabalhos têm demonstrado a necessidade da atividade física. Pessoas ativas têm um risco diminuído para o desenvolvimento de várias doenças 4,5,6. No 
Brasil, o sedentarismo apresenta alta prevalência, causando custos elevados, tanto diretos quanto indiretos, para o sistema de saúde 7,8. Diminuir o sedentarismo e promover estilos de vida mais saudáveis com a participação da atenção básica à saúde e seus profissionais pode representar um grande impacto na melhoria dos índices de saúde populacional e nos custos relacionados à gestão dos serviços 9,10,11.

Este estudo tem como objetivo descrever a prevalência de sedentarismo e fatores associados em populações das áreas de abrangência de unidades básicas de saúde em municípios das regiões Sul e Nordeste do Brasil.

\section{Métodos}

Um estudo de delineamento transversal selecionou uma amostra de indivíduos adultos (30-64 anos) e idosos (65 anos ou mais), residentes nas áreas de abrangência das unidades básicas de saúde, de 41 municípios com mais de 100 mil habitantes dos Estados do Rio Grande do Sul e Santa Catarina, na Região Sul; Alagoas, Pernambuco, Paraíba, Rio Grande do Norte e Piauí, na Região Nordeste do Brasil 12. Os municípios incluídos no estudo compõem os Lotes 2 Sul e Nordeste do Estudo de Linha de Base do Projeto de Expansão e Consolidação da Saúde da Família (PROESF) 3,12.

Uma amostra aleatória de 120 unidades básicas de saúde foi sorteada em cada um dos lotes estudados, com diferentes modalidades de atenção básica - Programa Saúde da Família (PSF) e tradicional. O sorteio das unidades foi proporcional ao tamanho da rede básica de cada município. Com base em listas produzidas pelos municípios, as unidades básicas de saúde foram selecionadas na razão de duas unidades com PSF para uma unidade tradicional. Quando o número de unidades básicas de saúde de um determinado modelo de atenção esgotava-se, complementava-se a amostra com unidades básicas de saúde com outra modalidade de atenção, garantindo representatividade da amostra em comparação à rede básica dos municípios. A seleção da amostra nos municípios foi proporcional à capacidade instalada de sua rede básica, pareando as unidades básicas de saúde por tamanho da área física. $\mathrm{Na}$ Região Sul, obteve-se uma amostra de 69 unidades básicas de saúde de PSF e 51 tradicionais. No Nordeste, a amostra foi constituída de 79 unidades básicas de saúde de PSF e 41 tradicionais. As unidades sorteadas orientaram a seleção da amostra de adultos e idosos da área de abrangência dos serviços.

Após a seleção das unidades básicas de saúde, a amostra foi selecionada de forma sistemática e independente para adultos e idosos, residentes na área de abrangência de cada unidade. Esses indivíduos foram localizados na área de abrangência de cada uma das unidades, ponto inicial para a amostragem sistemática. A estratégia para delimitação da área de abrangência incluiu a obtenção prévia do mapa da área de abrangência das unidades básicas de saúde, uma estimativa populacional valendo-se das áreas censitárias do IBGE e a localização dos indivíduos por meio de amostragem sistemática. Nos domicílios sorteados, apenas um morador era selecionado para participar do estudo. Para a aplicação do questionário, foram capacitados 15 supervisores de campo, que realizaram a coleta de dados após período de treinamento.

As amostras foram estimadas em 2.100 indivíduos por lote e grupo populacional, totalizando 4.200 indivíduos na Região Sul e 4.200 no Nordeste. Esse tamanho de amostra proporcionaria poder estatístico de, no mínimo, $80 \%$ para eventos com prevalência aproximada de $25 \%$. O número estimado de indivíduos para cada lote (2.100) foi dividido pelo número total de unidades básicas de saúde (120 unidades/lote), obtendo-se a necessidade de entrevistar 18 indivíduos na área de abrangência de cada uma das unidades 3 .

Com a amostra obtida de 4.060 adultos e de 4.003 idosos, a margem de erro para a estimativa de prevalência de sedentarismo foi de 1,5 ponto percentual. Para avaliação de associações, o estudo teve poder de $80 \%$ para detectar como significativas razões de prevalência de 1,2 ou maiores, para exposições que afetam de $10 \%$ a $90 \%$ da população, com nível de confiança de 95\%.

Unidades básicas tradicionais foram consideradas aquelas que se caracterizavam pela presença, na equipe, de médicos especialistas (clínico, pediatra e gineco-obstetra), enfermeiro, auxiliar de enfermagem e pessoal administrativo para apoio técnico. Muitas dessas unidades contavam com o apoio esporádico de outras especialidades profissionais (dentista, nutricionista, fisioterapeuta) e mesmo médicas. A população atendida caracteriza-se por ser espontânea ou encaminhada por outros serviços. As unidades de PSF, ao contrário, são formadas por equipes de saúde da família, que incluem médico generalista, enfermeiro, auxiliares de enfermagem e agentes comunitários, responsáveis pelo cadastramento e acompanhamento da população residente na área de abrangência do serviço 12 .

O nível de atividade física dos adultos e idosos foi avaliado utilizando-se a versão curta do Questionário Internacional de Atividade Física - (IPAQ) 13. Este instrumento avalia atividades físicas realizadas no tempo de lazer, como deslocamento de um lugar ao outro, serviços do- 
mésticos e atividades ocupacionais. Um escore de atividade física em minutos por semana foi construído, somando-se os minutos despendidos em caminhada e atividades de intensidade moderada com os minutos despendidos em atividades de intensidade vigorosa, multiplicados por dois. Tal estratégia visa a considerar as diferentes intensidades de cada atividade e está de acordo com as recomendações atuais quanto à prática de atividade física 7,14. Um escore abaixo de 150 minutos por semana foi o ponto de corte para classificar os indivíduos como sedentários. O questionário utilizado no estudo foi padronizado e pré-testado.

As variáveis independentes incluídas nesta análise foram: (a) sexo; (b) idade; (c) cor da pele (conforme a percepção do entrevistador); (d) escolaridade (anos de estudo com aprovação); (e) tabagismo (nunca fumou, ex-fumante, fumante atual); (f) padrão de consumo sócio-econômico (classificação da Associação Brasileira de Empresas de Pesquisa); (g) situação conjugal; (h) renda familiar per capita (em salários mínimos - SM); (i) trabalho remunerado no último mês (não/licença ou nunca trabalhou; sim); (j) autopercepção de saúde; (k) identificação da atividade física como um dos três fatores mais importantes para a manutenção da saúde; (l) prescrição de atividade física em consulta em unidade básica de saúde no último ano (não e sim).

As análises descritivas incluíram cálculos de proporções e respectivos intervalos de confiança de 95\% (IC95\%). Na análise bruta, a prevalência de sedentarismo foi calculada para cada grupo das variáveis independentes, e o nível de significância foi testado por meio dos testes de Wald para heterogeneidade e tendência linear. A análise ajustada foi realizada por regressão de Poisson 15 com cálculo de razões de prevalência ajustadas, IC95\% e valores de significância aferidos pelos mesmos testes descritos anteriormente. Todas as análises levaram em consideração o desenho amostral, e foi utilizado um modelo hierárquico de determinação do desfecho 16. O modelo incluiu as variáveis independentes "a- $h$ " no primeiro nível. A variável "i" foi incluída no segundo nível, enquanto o terceiro nível incluiu as variáveis " $\mathrm{j}$ - $\mathrm{k}$ ” e o quarto incluiu a variável independente "l". As análises foram realizadas no pacote estatístico Stata 9.2 (Stata Corp., College Station, Estados Unidos).

O Comitê de Ética da Faculdade de Medicina da Universidade Federal de Pelotas aprovou o protocolo do estudo, e obteve-se consentimento informado de todos os participantes. Dados detalhados sobre a metodologia do projeto estão disponíveis em outras publicações 3,12.

\section{Resultados}

Foi estimada uma amostra de 2.100 indivíduos em cada um dos quatro grupos estudados: (a) adultos da Região Sul; (b) idosos da Região Sul; (c) adultos da Região Nordeste; (d) idosos da Região Nordeste. No total, foram realizadas 4.060 entrevistas com adultos e 4003 com idosos, resultando em taxas de não-resposta de 3,5\% e 4,7\%, respectivamente. A prevalência de sedentarismo somando-se as duas regiões foi de 31,8\% (IC95\%: 30,4-33,2) em adultos e de 58\% (IC95\%: 56,4$59,5)$ em idosos. A prevalência de sedentarismo foi significativamente maior na Região Nordeste, para ambas as faixas etárias (Figura 1).

A Tabela 1 descreve a amostra de adultos e idosos conforme as variáveis independentes incluídas na investigação. A proporção de mulheres foi maior em ambos os grupos etários, embora a diferença seja mais marcante entre os idosos. Entre os adultos, a média de idade foi de 45,5 anos (desvio padrão - DP = 9,6), enquanto entre os idosos a média foi de 73,9 anos (DP = 7,0 ). Cerca de dois terços dos indivíduos nos dois grupos etários foram classificados como brancos, em termos de cor de pele. A escolaridade foi claramente superior entre os adultos em comparação aos idosos. A prevalência de tabagismo atual foi de $27,7 \%$ nos adultos e $15,2 \%$ nos ido-

Figura 1

Prevalência de sedentarismo em adultos e idosos na população das áreas de abrangência de unidades básicas de saúde de municípios das regiões Sul e Nordeste do Brasil, 2005.

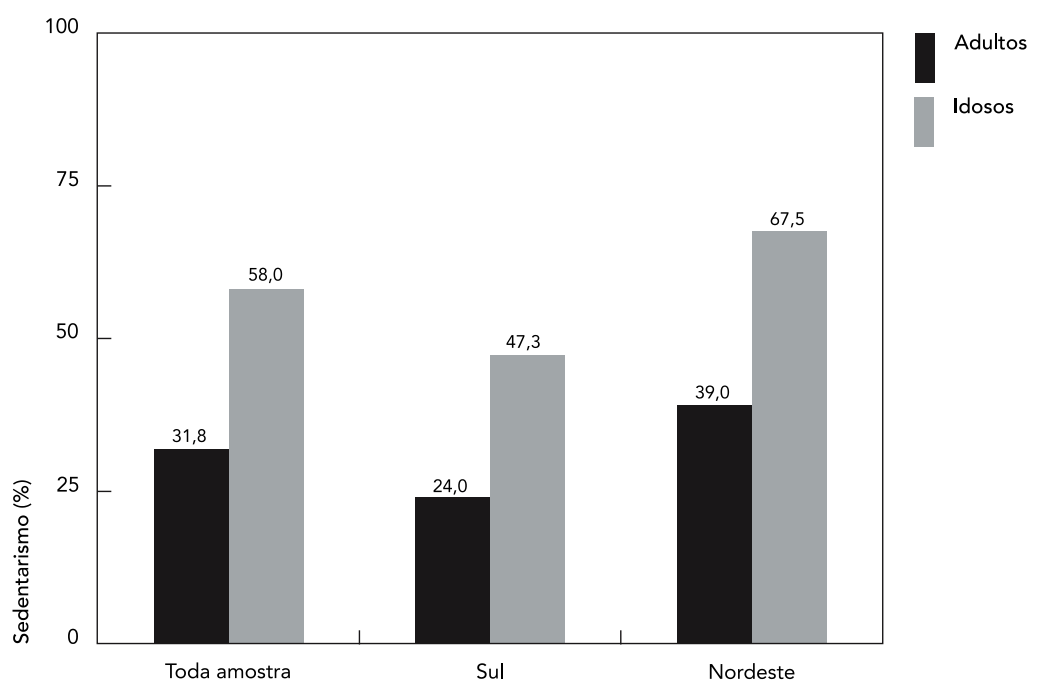


Tabela 1

Descrição das variáveis independentes em adultos e idosos na população das áreas de abrangência de unidades básicas de saúde de municípios das regiões Sul e Nordeste do Brasil, 2005.

\begin{tabular}{|c|c|c|c|c|}
\hline Variáveis & Adultos (\%) & $\mathbf{N}$ & Idosos (\%) & $\mathbf{N}$ \\
\hline Sexo & & 4.060 & & 4.003 \\
\hline Masculino & 44,9 & & 38,8 & \\
\hline Feminino & 55,1 & & 61,9 & \\
\hline Idade (anos) & & 4.059 & & 4.003 \\
\hline $30-40$ & 36,2 & & - & \\
\hline $41-50$ & 31,1 & & - & \\
\hline $51-64$ & 32,6 & & - & \\
\hline $65-70$ & - & & 38,5 & \\
\hline $71-75$ & - & & 25,7 & \\
\hline $76-79$ & - & & 18,5 & \\
\hline 80 ou + & - & & 17,3 & \\
\hline Cor da pele & & 4.039 & & 3.985 \\
\hline Branca & 65,3 & & 70,0 & \\
\hline Não branca & 34,7 & & 30,0 & \\
\hline Escolaridade * & & 4.047 & & 3.923 \\
\hline Não estudou & 16,7 & & 49,4 & \\
\hline Fundamental incompleto & 47,6 & & 43,2 & \\
\hline Fundamental completo & 11,5 & & & \\
\hline Médio incompleto & 4,9 & & 4,00 & \\
\hline Médio completo & 14,9 & & & \\
\hline Superior & 4,4 & & 3,5 & \\
\hline Tabagismo & & 4.060 & & 4.000 \\
\hline Não, nunca fumou & 45,9 & & 43,4 & \\
\hline Ex-fumante & 26,4 & & 41,5 & \\
\hline Fumante & 27,7 & & 15,2 & \\
\hline Padrão de consumo sócio-econômico & & 3.908 & & 3.650 \\
\hline A, B ou C & 37,4 & & 21,0 & \\
\hline $\mathrm{D}$ & 32,4 & & 33,7 & \\
\hline $\mathrm{E}$ & 30,1 & & 45,3 & \\
\hline Situação conjugal & & 4.060 & & 3.970 \\
\hline Casado(a)/Com companheiro(a) & 73,3 & & 42,7 & \\
\hline Viúvo & 7,1 & & 45,0 & \\
\hline Separado(a)/Divorciado(a) & 9,9 & & 7,8 & \\
\hline Solteiro(a) & 9,8 & & - & \\
\hline Renda familiar per capita (salários mínimos) & & 4.046 & & 3.990 \\
\hline Sem renda & 7,8 & & 3,9 & \\
\hline Até 1 & 66,7 & & 74,6 & \\
\hline $1,01-2$ & 17,6 & & 15,4 & \\
\hline $2,01-3$ & 4,5 & & 3,7 & \\
\hline 3 ou + & 3,5 & & 2,5 & \\
\hline Trabalho remunerado no último mês & & 4.056 & & 3.957 \\
\hline Não & 51,3 & & 94,7 & \\
\hline Sim & 48,7 & & 5,3 & \\
\hline Autopercepção da saúde & & 4.048 & & 3.951 \\
\hline Excelente & 6,7 & & 2,6 & \\
\hline Muito boa & 6,3 & & 3,2 & \\
\hline Boa & 38,3 & & 29,7 & \\
\hline Regular & 38,2 & & 43,8 & \\
\hline Ruim & 10,5 & & 20,7 & \\
\hline
\end{tabular}

(continua) 


\begin{tabular}{|c|c|c|c|c|}
\hline Variáveis & Adultos (\%) & $\mathbf{N}$ & Idosos (\%) & $\mathbf{N}$ \\
\hline Identificação de atividade física como & & 4.036 & & 3.660 \\
\hline \multicolumn{5}{|l|}{ benefício para saúde ** } \\
\hline Não & 39,0 & & 26,8 & \\
\hline $\operatorname{Sim}$ & 61,0 & & 73,8 & \\
\hline Prescrição de atividade física em unidade & & 3.278 & & 3.338 \\
\hline \multicolumn{5}{|l|}{ básica de saúde no último ano } \\
\hline Não & 23,9 & & 69,7 & \\
\hline Sim & 76,1 & & 30,3 & \\
\hline
\end{tabular}

sos, os quais tiveram um percentual elevado de ex-fumantes $(41,5 \%)$. A maioria dos adultos era casada ou vivia com companheiro $(73,3 \%)$, enquanto no grupo de idosos a categoria de maior prevalência foi a de viúvos (45\%). Seja na amostra de adultos, seja na de idosos, a categoria de renda familiar per capita de até um salário mínimo foi a de maior prevalência. Aproximadamente metade da população de adultos trabalhou com remuneração no último mês, enquanto para o grupo de idosos esse percentual foi de apenas 5,3\%. Cerca de dois terços dos idosos classificou sua saúde como regular ou ruim, ao passo que, entre os adultos, esse percentual foi próximo de $50 \%$. O percentual de indivíduos que classificou a atividade física como um dos três comportamentos mais importantes para a manutenção da saúde foi de $61 \%$ entre os adultos e $74 \%$ entre os idosos. No primeiro grupo, $76,1 \%$ receberam prescrição de atividade física na unidade básica de saúde no último ano, enquanto, no segundo, esse valor foi de apenas $30,3 \%$.

A Tabela 2 apresenta a associação bruta e ajustada entre sedentarismo e as variáveis independentes, combinando-se os dados dos municípios das regiões Sul e Nordeste. O sedentarismo foi significativamente mais freqüente entre os homens em comparação às mulheres, tanto na análise bruta, quanto na análise ajustada, sendo as diferenças marcantes entre os adultos e de pequena magnitude entre os idosos. Para ambos os grupos, o sedentarismo tendeu a crescer com o aumento da idade. Embora estatisticamente significativas, as diferenças relacionadas à cor da pele entre os idosos foram de magnitude pequena. A escolaridade não se mostrou associada ao sedentarismo em adultos e mostrou uma relação de pequena magnitude entre os idosos após ajuste. As variáveis tabagismo, padrão de consumo sócio-econômico e situação conjugal não se mostraram associadas ao sedentarismo nas análises ajustadas. A baixa renda familiar apresentou-se como fator de risco para sedentarismo tanto em adultos, quanto em idosos. As pessoas que trabalham com remuneração apresentaram menor risco de sedentarismo em ambos os grupos etários. A autopercepção de saúde ruim esteve fortemente associada com o sedentarismo em ambos as faixas etárias. $\mathrm{O}$ fato de as pessoas identificarem ou não a prática de atividade física como um dos fatores mais importantes para a manutenção da saúde não se associou com a prevalência de sedentarismo. Da mesma forma, as pessoas que receberam prescrição para prática de atividade física em unidade básica de saúde no último ano não apresentaram prevalência de sedentarismo, diferentemente daquelas que não receberam tal prescrição.

As Tabelas 3 e 4 repetem as análises separadamente para as regiões Sul e Nordeste. Destacaremos nesta seção apenas as diferenças encontradas nos fatores de risco para sedentarismo entre as duas regiões. Em relação à variável sexo, os homens idosos apresentaram percentual significativamente maior de sedentarismo quando comparados às mulheres somente no Sul. Nesta região, a idade não se associou com o sedentarismo entre os adultos, porém foi determinante do sedentarismo em adultos do Nordeste. A renda familiar per capita foi um importante fator associado ao sedentarismo entre idosos do Sul, mas a mesma associação não foi detectada no Nordeste. O trabalho remunerado foi fator de proteção para sedentarismo em idosos da Região Sul, o que não ocorreu no Nordeste. Entre os adultos do Nordeste, aqueles que identificaram a atividade física como um dos fatores mais importantes para a manutenção da saúde foram os que apresentaram risco elevado para sedentarismo. Nos idosos do Sul, aqueles que receberam pres- 
Prevalência, análise bruta e ajustada para adultos e idosos em relação ao desfecho sedentarismo na população da área de abrangência de unidades básicas de saúde de estados do Sul e Nordeste do Brasil.

\begin{tabular}{|c|c|c|c|c|c|}
\hline \multirow[t]{2}{*}{ Variáveis (nível) * } & \multirow[b]{2}{*}{$\%$} & \multicolumn{2}{|c|}{ Análise bruta } & \multicolumn{2}{|c|}{ Análise ajustada } \\
\hline & & RP (IC95\%) & $\mathrm{p}$ & RP (IC95\%) & $p$ \\
\hline \multicolumn{6}{|l|}{ Adultos } \\
\hline Sexo (1) & & & $<0,001$ & & $<0,001$ \\
\hline Masculino & 37,5 & 1,00 & & 1,00 & \\
\hline Feminino & 27,2 & $0,72(0,66-0,79)$ & & $0,72(0,66-0,79)$ & \\
\hline Idade (anos) (1) & & & 0,02 ** & & 0,03 ** \\
\hline $30-40$ & 30,5 & 1,00 & & 1,00 & \\
\hline $41-50$ & 30,2 & $0,99(0,88-1,11)$ & & $0,99(0,89-1,11)$ & \\
\hline $51-64$ & 34,7 & $1,13(1,02-1,26)$ & & $1,12(1,00-1,24)$ & \\
\hline \multicolumn{6}{|l|}{$65-70$} \\
\hline \multicolumn{6}{|l|}{$71-75$} \\
\hline \multicolumn{6}{|l|}{$76-79$} \\
\hline \multicolumn{6}{|l|}{80 ou +} \\
\hline Cor de pele (1) & & & 0,23 & & 0,24 \\
\hline Branca & 32,4 & 1,00 & & 1,00 & \\
\hline Não Branca & 30,6 & $0,94(0,86-1,04)$ & & $0,94(0,85-1,03)$ & \\
\hline 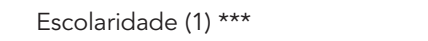 & & & 0,16 ** & & 0,89 ** \\
\hline Não estudou & 37,8 & 1,00 & & 1,00 & \\
\hline Fundamental incompleto & 47,6 & $0,81(0,72-0,91)$ & & $0,82(0,72-0,93)$ & \\
\hline Fundamental completo & 30,7 & $0,83(0,69-0,96)$ & & $0,86(0,71-1,04)$ & \\
\hline Médio incompleto & 28,6 & $0,76(0,60-0,96)$ & & $0,79(0,61-1,03)$ & \\
\hline Médio completo & 30,4 & $0,80(0,69-0,94)$ & & $0,87(0,72-1,05)$ & \\
\hline Superior & 35 & $0,93(0,74-1,15)$ & & $1,06(0,82-1,37)$ & \\
\hline Tabagismo (1) & & & 0,96 & & 0,1 \\
\hline Não, nunca fumou & 30,8 & 1,00 & & 1,00 & \\
\hline Já fumou & 35,1 & $1,13(1,02-1,27)$ & & $1,05(0,95-1,18)$ & \\
\hline Fumante atual & 30,3 & $0,98(0,87-1,10)$ & & $0,92(0,83-1,04)$ & \\
\hline \multicolumn{2}{|c|}{ Padrão de consumo sócio-econômico (1) } & & 0,17 ** & & 0,21 ** \\
\hline A, B ou C & 30,4 & 1,00 & & 1,00 & \\
\hline $\mathrm{D}$ & 31,4 & $1,04(0,92-1,16)$ & & $1,02(0,91-1,15)$ & \\
\hline E & 33 & $1,09(0,97-1,22)$ & & $1,09(0,96-1,23)$ & \\
\hline \multicolumn{2}{|l|}{ Situação conjugal (1) } & & 0,53 & & 0,98 \\
\hline Casado(a)/Com companheiro(a) & 32,2 & 1,00 & & 1,00 & \\
\hline Viúvo(a) & 30,3 & $0,94(0,78-1,13)$ & & $1,01(0,83-1,23)$ & \\
\hline Separado(a)/Divorciado(a) & 29,6 & $0,92(0,78-1,08)$ & & $0,96(0,82-1,14)$ & \\
\hline Solteiro(a) & 32,1 & $1,00(0,85-1,16)$ & & $1,02(0,87-1,19)$ & \\
\hline \multicolumn{2}{|c|}{ Renda familiar per capita (salários mínimos) (1) } & & 0,13 & & $0,04 * *$ \\
\hline Sem renda & 41 & 1,00 & & 1,00 & \\
\hline Até 1 & 32 & $0,76(0,66-0,88)$ & & $0,77(0,67-0,89)$ & \\
\hline $1,01-2$ & 29,8 & $0,73(0,61-0,86)$ & & $0,70(0,59-0,84)$ & \\
\hline $2,01-3$ & 30,6 & $0,74(0,57-0,96)$ & & $0,72(0,56-0,93)$ & \\
\hline $3 \mathrm{ou}+$ & 34,3 & $0,84(0,64-1,09)$ & & $0,80(0,61-1,05)$ & \\
\hline \multicolumn{2}{|l|}{ Trabalho remunerado no último mês (2) } & & 0,02 & & $<0,001$ \\
\hline Não, licença ou nunca trabalhou & 33,5 & 1,00 & & 1,00 & \\
\hline Sim & 30,1 & $0,90(0,82-0,98)$ & & $0,82(0,75-0,90)$ & \\
\hline \multicolumn{2}{|l|}{ Autopercepção da saúde (3) } & & $<0,001 * \star$ & & $<0,001$ \\
\hline Excelente & 27,8 & 1,00 & & 1,00 & \\
\hline Muito boa & 27,2 & $0,97(0,74-1,30)$ & & $1,04(0,79-1,38)$ & \\
\hline Boa & 29,3 & $1,05(0,86-1,30)$ & & $1,12(0,90-1,38)$ & \\
\hline Regular & 32,9 & $1,18(0,96-1,45)$ & & $1,25(1,01-1,55)$ & \\
\hline Ruim & 42,4 & $1,53(1,22-1,91)$ & & $1,60(1,28-2,02)$ & \\
\hline
\end{tabular}

(continua) 


\begin{tabular}{|c|c|c|c|c|c|}
\hline \multirow[t]{2}{*}{ Variáveis (nível) * } & \multirow[b]{2}{*}{$\%$} & \multicolumn{2}{|c|}{ Análise bruta } & \multicolumn{2}{|c|}{ Análise ajustada } \\
\hline & & RP (IC95\%) & $p$ & RP (IC95\%) & $p$ \\
\hline Identificação da atividade física como & & & 0,47 & & 0,6 \\
\hline \multicolumn{6}{|l|}{ benefício para saúde (3) } \\
\hline Não & 31,0 & 1,00 & & 1,00 & \\
\hline $\operatorname{Sim}$ & 32,1 & $1,04(0,94-1,14)$ & & $1,03(0,93-1,13)$ & \\
\hline \multicolumn{2}{|c|}{ Prescrição de atividade física no último ano (4) } & & 0,37 & & 0,65 \\
\hline Não & 31,0 & 1,00 & & 1,00 & \\
\hline Sim & 32,7 & $1,05(0,94-1,19)$ & & $1,03(0,91-1,16)$ & \\
\hline \multicolumn{6}{|l|}{ Idosos } \\
\hline Sexo (1) & & & 0,09 & & 0,007 \\
\hline Masculino & 59,6 & 1,00 & & 1,00 & \\
\hline Feminino & 56,9 & $0,95(0,90-1,00)$ & & $0,93(0,88-0,98)$ & \\
\hline Idade (anos) (1) & & & $<0,001$ ** & & $<0,001 * *$ \\
\hline \multicolumn{6}{|l|}{$30-40$} \\
\hline \multicolumn{6}{|l|}{$41-50$} \\
\hline \multicolumn{6}{|l|}{$51-64$} \\
\hline $65-70$ & 47,0 & 1,00 & & 1,00 & \\
\hline $71-75$ & 56,2 & $1,20(1,11-1,29)$ & & $1,19(1,10-1,29)$ & \\
\hline $76-79$ & 64,7 & $1,38(1,28-1,48)$ & & $1,36(1,26-1,47)$ & \\
\hline $80 \mathrm{ou}+$ & 77,9 & $1,66(1,55-1,77)$ & & $1,63(1,52-1,74)$ & \\
\hline Cor de pele (1) & & & 0,001 & & 0,03 \\
\hline Branca & 56,3 & 1,00 & & 1,00 & \\
\hline Não branca & 61,9 & $1,10(1,04-1,16)$ & & $1,05(1,00-1,12)$ & \\
\hline 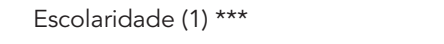 & & & $<0,001$ & & 0,002 \\
\hline Não estudou & 63,8 & 1,00 & & 1,00 & \\
\hline Fundamental incompleto & 52,7 & $0,83(0,78-0,87)$ & & $0,89(0,84-0,94)$ & \\
\hline \multicolumn{6}{|l|}{ Fundamental completo } \\
\hline Médio incompleto & 46,1 & $0,72(0,61-0,86)$ & & $0,85(0,71-1,01)$ & \\
\hline \multicolumn{6}{|l|}{ Médio completo } \\
\hline Superior & 50 & $0,78(0,66-0,93)$ & & $0,95(0,79-1,13)$ & \\
\hline Tabagismo (1) & & & $<0,001$ & & 0,81 \\
\hline Não, nunca fumou & 55,6 & 1,00 & & 1,00 & \\
\hline Já fumou & 62 & $1,12(1,05-1,18)$ & & $1,08(1,02-1,15)$ & \\
\hline Fumante atual & 54 & $0,97(0,89-1,06)$ & & $0,97(0,89-1,06)$ & \\
\hline \multicolumn{2}{|l|}{ Padrão de consumo sócio-econômico (1) } & & $<0,001$ & & 0,65 \\
\hline A, B ou C & 52,7 & 1,00 & & 1,00 & \\
\hline D & 58,3 & $1,11(1,02-1,20)$ & & $1,04(0,96-1,14)$ & \\
\hline E & 61 & $1,16(1,07-1,26)$ & & $1,03(0,95-1,12)$ & \\
\hline \multicolumn{2}{|l|}{ Situação conjugal (1) } & & 0,1 & & 0,31 \\
\hline Casado(a)/Com companheiro(a) & 54,7 & 1,00 & & 1,00 & \\
\hline Viúvo(a) & 62,2 & $1,14(1,08-1,21)$ & & $1,10(1,03-1,17)$ & \\
\hline Separado(a)/Divorciado(a) & 52,5 & $0,96(0,86-1,08)$ & & $1,00(0,89-1,12)$ & \\
\hline Solteiro(a) & 57,6 & $1,05(0,92-1,20)$ & & $1,05(0,92-1,21)$ & \\
\hline \multicolumn{2}{|c|}{ Renda familiar per capita (salários mínimos) (1) } & & $<0,001$ ** & & $<0,001 * \star$ \\
\hline Sem renda & 60,1 & 1,00 & & 1,00 & \\
\hline Até 1 & 60,3 & $1,00(0,88-1,14)$ & & $0,98(0,85-1,12)$ & \\
\hline $1,01-2$ & 52,3 & $0,87(0,75-1,00)$ & & $0,89(0,76-1,03)$ & \\
\hline $2,01-3$ & 37,4 & $0,62(0,49-0,80)$ & & $0,63(0,49-0,81)$ & \\
\hline 3 ou + & 50 & $0,83(0,66-1,06)$ & & $0,85(0,67-1,07)$ & \\
\hline Trabalho remunerado no último mês & & & $<0,001$ & & $<0,001$ \\
\hline Não, licença ou nunca trabalhou & 59,1 & 1,00 & & 1,00 & \\
\hline $\operatorname{Sim}$ & 39,9 & $0,67(0,57-0,80)$ & & $0,74(0,63-0,88)$ & \\
\hline
\end{tabular}

(continua) 


\begin{tabular}{|c|c|c|c|c|c|}
\hline \multirow[t]{2}{*}{ Variáveis (nível) * } & \multirow[b]{2}{*}{$\%$} & \multicolumn{2}{|c|}{ Análise bruta } & \multicolumn{2}{|c|}{ Análise ajustada } \\
\hline & & RP (IC95\%) & $\mathrm{p}$ & RP (IC95\%) & $\mathrm{p}$ \\
\hline Autopercepção da saúde (3) & & & $<0,001 \star \star$ & & $<0,001 * *$ \\
\hline Excelente & 41,4 & 1,00 & & 1,00 & \\
\hline Muito boa & 37,4 & $0,90(0,65-1,25)$ & & $0,87(0,63-1,21)$ & \\
\hline Boa & 50,2 & $1,21(0,96-1,54)$ & & $1,18(0,93-1,49)$ & \\
\hline Regular & 58,6 & $1,41(1,12-1,79)$ & & $1,36(1,08-1,71)$ & \\
\hline Ruim & 71,7 & $1,73(1,37-2,19)$ & & $1,62(1,28-2,04)$ & \\
\hline Identificação da atividade física como & & & 0,72 & & 0,42 \\
\hline \multicolumn{6}{|l|}{ benefício para saúde (3) } \\
\hline Não & 56,1 & 1,00 & & 1,00 & \\
\hline Sim & 55,4 & $0,99(0,92-1,06)$ & & $0,97(0,91-1,04)$ & \\
\hline \multicolumn{2}{|c|}{ Prescrição de atividade física no último ano (4) } & & 0,003 & & 0,11 \\
\hline Não & 59,3 & 1,00 & & 1,00 & \\
\hline Sim & 53,7 & $0,90(0,85-0,97)$ & & $0,95(0,89-1,01)$ & \\
\hline
\end{tabular}

* Corresponde ao nível da variável no modelo hierárquico - cada associação foi ajustada para as variáveis do mesmo nível ou de nível superior no modelo;

** Teste de tendência;

*** Para idosos, estão agrupadas as categorias da variável escolaridade: fundamental completo e ensino médio incompleto, assim como médio completo e superior.

Tabela 3

Prevalência de sedentarismo, análise bruta e ajustada para adultos e idosos na população da área de abrangência de unidades básicas de saúde de municípios da Região Sul do Brasil, 2005.

\begin{tabular}{|c|c|c|c|c|c|}
\hline \multirow[t]{2}{*}{ Variáveis (nível) * } & \multirow[b]{2}{*}{$\%$} & \multicolumn{2}{|c|}{ Análise bruta } & \multicolumn{2}{|c|}{ Análise ajustada } \\
\hline & & RP (IC95\%) & $p$ & RP (IC95\%) & $p$ \\
\hline \multicolumn{6}{|l|}{ Adultos } \\
\hline Sexo (1) & & & $<0,001$ & & $<0,001$ \\
\hline Masculino & 30,15 & 1,00 & & 1,00 & \\
\hline Feminino & 19,50 & $0,64(0,55-0,76)$ & & $0,65(0,55-0,76)$ & \\
\hline Idade (anos) (1) & & & $0,79 * *$ & & $0,71 * *$ \\
\hline $30-40$ & 24,37 & 1,00 & & 1,00 & \\
\hline $41-50$ & 23,90 & $0,98(0,81-1,19)$ & & $0,97(0,80-1,18)$ & \\
\hline $51-64$ & 23,75 & $0,97(0,80-1,18)$ & & $0,96(0,80-1,17)$ & \\
\hline \multicolumn{6}{|l|}{$65-70$} \\
\hline \multicolumn{6}{|l|}{$71-75$} \\
\hline \multicolumn{6}{|l|}{$76-79$} \\
\hline \multicolumn{6}{|l|}{80 ou +} \\
\hline Cor de pele (1) & & & 0,007 & & 0,01 \\
\hline Branca & 25,39 & 1,00 & & 1,00 & \\
\hline Não branca & 18,64 & $0,73(0,59-0,92)$ & & $0,75(0,60-0,94)$ & \\
\hline Escolaridade (1) *** & & & 0,63 ** & & 0,92 ** \\
\hline Não estudou & 27,11 & 1,00 & & 1,00 & \\
\hline Fundamental incompleto & 23,11 & $0,85(0,65-1,12)$ & & $0,81(0,62-1,08)$ & \\
\hline Fundamental completo & 24,91 & $0,92(0,66-1,27)$ & & $0,87(0,62-1,21)$ & \\
\hline Médio incompleto & 19,59 & $0,72(0,45-1,16)$ & & $0,63(0,39-1,63)$ & \\
\hline Médio completo & 24,44 & $0,90(0,65-1,25)$ & & $0,84(0,59-1,18)$ & \\
\hline Superior & 29,47 & $1,09(0,73-1,62)$ & & $1,04(0,68-1,58)$ & \\
\hline Tabagismo (1) & & & 0,01 & & 0,52 \\
\hline Não, nunca fumou & 21,6 & 1,00 & & 1,00 & \\
\hline Já fumou & 28,75 & $1,33(1,10-1,61)$ & & $1,24(1,02-1,51)$ & \\
\hline Fumante atual & 23,44 & $1,09(0,90-1,32)$ & & $1,06(0,87-1,28)$ & \\
\hline
\end{tabular}

(continua) 


\begin{tabular}{|c|c|c|c|c|c|}
\hline \multirow[t]{2}{*}{ Variáveis (nível) * } & \multirow[b]{2}{*}{$\%$} & \multicolumn{2}{|c|}{ Análise bruta } & \multicolumn{2}{|c|}{ Análise ajustada } \\
\hline & & RP (IC95\%) & $\mathrm{p}$ & RP (IC95\%) & $\mathrm{p}$ \\
\hline \multicolumn{2}{|l|}{ Padrão de consumo sócio-econômico (1) } & & 0,46 ** & & 0,96 \\
\hline A, B ou C & 24,35 & 1,00 & & 1,00 & \\
\hline $\mathrm{D}$ & 24,32 & $1,00(0,83-1,20)$ & & $1,02(0,84-1,25)$ & \\
\hline$E$ & 21,58 & $0,87(0,70-1,12)$ & & $0,99(0,76-1,29)$ & \\
\hline \multicolumn{2}{|l|}{ Situação conjugal (1) } & & 0,28 & & 0,98 \\
\hline Casado(a)/Com companheiro(a) & 25,07 & 1,00 & & 1,00 & \\
\hline Viúvo(a) & 22,08 & $0,88(0,65-1,20)$ & & $1,05(0,77-1,44)$ & \\
\hline Separado(a)/Divorciado(a) & 21,99 & $0,88(0,66-1,16)$ & & $0,96(0,72-1,27)$ & \\
\hline Solteiro(a) & 18,75 & $0,75(0,54-1,05)$ & & $0,782(0,56-1,09)$ & \\
\hline \multicolumn{2}{|c|}{ Renda familiar per capita (salário mínimo) (1) } & & 0,83 ** & & 0,76 ** \\
\hline Sem renda & 30,00 & 1,00 & & 1,00 & \\
\hline Até 1 & 22,82 & $0,76(0,57-1,02)$ & & $0,74(0,55-1,00)$ & \\
\hline $1,01-2$ & 24,63 & $0,82(0,60-1,13)$ & & $0,77(0,55-1,06)$ & \\
\hline $2,01-3$ & 26,72 & $0,89(0,60-1,32)$ & & $0,81(0,55-1,22)$ & \\
\hline 3 ou + & 24,49 & $0,82(0,52-1,27)$ & & $0,77(0,49-1,20)$ & \\
\hline \multicolumn{2}{|l|}{ Trabalho remunerado no último mês (2) } & & 0,44 & & 0,02 \\
\hline Não, licença ou nunca trabalhou & 24,79 & 1,00 & & 1,00 & \\
\hline $\operatorname{Sim}$ & 23,29 & $0,94(0,80-1,10)$ & & $0,82(0,70-0,97)$ & \\
\hline \multicolumn{2}{|l|}{ Autopercepção da saúde (3) } & & 0,08 ** & & 0,03 ** \\
\hline Excelente & 21,62 & 1,00 & & 1,00 & \\
\hline Muito boa & 22,88 & $1,06(0,69-1,61)$ & & $1,08(0,70-1,64)$ & \\
\hline Boa & 23,54 & $1,09(0,78-1,51)$ & & $1,15(0,82-1,61)$ & \\
\hline Regular & 22,96 & $1,06(0,76-1,49)$ & & $1,14(0,81-1,61)$ & \\
\hline Ruim & 33,75 & $1,56(1,07-2,27)$ & & $1,65(1,13-2,40)$ & \\
\hline Identificação da atividade física como & 0,89 & & 0,56 & & \\
\hline \multicolumn{6}{|l|}{ benefício para saúde (3) \# } \\
\hline Não & 24,20 & 1,00 & & 1,00 & \\
\hline Sim & 23,80 & $0,99(0,83-1,18)$ & & $1,05(0,88-1,26)$ & \\
\hline \multicolumn{2}{|c|}{ Prescrição de atividade física no último ano (4) } & & 0,87 & & 0,87 \\
\hline Não & 23,51 & 1,00 & & 1,00 & \\
\hline Sim & 23,05 & $0,98(0,78-1,23)$ & & $0,98(0,78-1,23)$ & \\
\hline \multicolumn{6}{|l|}{ Idosos } \\
\hline \multicolumn{2}{|l|}{ Sexo (1) } & & 0,1 & & 0,005 \\
\hline Masculino & 49,78 & 1,00 & & 1,00 & \\
\hline Feminino & 45,84 & $0,92(0,84-1,01)$ & & $0,87(0,79-0,96)$ & \\
\hline \multicolumn{2}{|l|}{ Idade (anos) (1) } & & $<0,001$ ** & & $<0,001 * \star$ \\
\hline \multicolumn{6}{|l|}{$30-40$} \\
\hline \multicolumn{6}{|l|}{$41-50$} \\
\hline \multicolumn{6}{|l|}{$51-64$} \\
\hline $65-70$ & 36,79 & 1,00 & & 1,00 & \\
\hline $71-75$ & 45,82 & $1,25(1,09-1,43)$ & & $1,26(1,10-1,44)$ & \\
\hline $76-79$ & 53,67 & $1,46(1,27-1,67)$ & & $1,46(1,27-1,68)$ & \\
\hline 80 ou + & 69,44 & $1,89(1,67-2,13)$ & & $1,90(1,68-2,15)$ & \\
\hline Cor de pele (1) & & & 0,94 & & 0,29 \\
\hline Branca & 47,37 & 1,00 & & 1,00 & \\
\hline Não branca & 47,15 & $0,99(0,88-1,13)$ & & $0,93(0,82-1,06)$ & \\
\hline 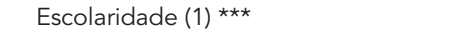 & & & $<0,001$ & & 0,06 \\
\hline Não estudou & 52,23 & 1,00 & & 1,00 & \\
\hline Fundamental incompleto & 45,60 & $0,87(0,79-0,96)$ & & $0,94(0,85-1,04)$ & \\
\hline \multicolumn{6}{|l|}{ Fundamental completo } \\
\hline Médio incompleto & 34,48 & $0,66(0,50-0,89)$ & & $0,76(0,57-1,01)$ & \\
\hline \multicolumn{6}{|l|}{ Médio completo } \\
\hline Superior & 37,50 & $0,72(0,52-0,99)$ & & $0,87(0,63-1,21)$ & \\
\hline
\end{tabular}

(continua) 


\begin{tabular}{|c|c|c|c|c|c|}
\hline \multirow[t]{2}{*}{ Variáveis (nível) * } & \multirow[b]{2}{*}{$\%$} & \multicolumn{2}{|c|}{ Análise bruta } & \multicolumn{2}{|c|}{ Análise ajustada } \\
\hline & & RP (IC95\%) & $\mathrm{p}$ & RP (IC95\%) & $\mathrm{p}$ \\
\hline \multicolumn{2}{|l|}{ Tabagismo (1) } & & 0,94 & & 0,53 \\
\hline Não, nunca fumou & 45,84 & 1,00 & & 1,00 & \\
\hline Já fumou & 50,95 & $1,11(1,00-1,23)$ & & $1,14(1,02-1,27)$ & \\
\hline Fumante atual & 43,01 & $0,94(0,81-1,09)$ & & $1,00(0,85-1,17)$ & \\
\hline \multicolumn{2}{|c|}{ Padrão de consumo sócio-econômico (1) } & & 0,08 & & 0,94 \\
\hline A, B ou C & 42,53 & 1,00 & & 1,00 & \\
\hline $\mathrm{D}$ & 51,32 & $1,20(1,06-1,37)$ & & $1,15(1,01-1,31)$ & \\
\hline$E$ & 48,25 & $1,13(0,99-1,30)$ & & $1,02(0,89-1,18)$ & \\
\hline \multicolumn{2}{|l|}{ Situação conjugal (1) } & & 0,93 & & 0,93 \\
\hline Casado(a)/Com companheiro(a) & 44,77 & 1,00 & & 1,00 & \\
\hline Viúvo(a) & 51,41 & $1,15(1,04-1,27)$ & & $1,13(1,00-1,28)$ & \\
\hline Separado(a)/Divorciado (a) & 39,86 & $0,89(0,71-1,11)$ & & $0,97(0,78-1,21)$ & \\
\hline Solteiro(a) & 41,18 & $0,92(0,69-1,23)$ & & $0,91(0,67-1,23)$ & \\
\hline \multicolumn{2}{|c|}{ Renda familiar per capita (salário mínimo) (1) } & & $<0,001$ ** & & 0,001 ** \\
\hline Sem renda & 48,48 & 1,00 & & 1,00 & \\
\hline Até 1 & 50,37 & $1,04(0,81-1,34)$ & & $1,06(0,82-1,39)$ & \\
\hline $1,01-2$ & 44,20 & $0,91(0,69-1,20)$ & & $0,96(0,73-1,28)$ & \\
\hline $2,01-3$ & 29,63 & $0,61(0,42-0,90)$ & & $0,64(0,43-0,95)$ & \\
\hline 3 ou + & 36,92 & $0,76(0,51-1,14)$ & & $0,80(0,54-1,21)$ & \\
\hline \multicolumn{2}{|l|}{ Trabalho remunerado no último mês (2) } & & $<0,001$ & & 0,002 \\
\hline Não, licença ou nunca trabalhou & 48,80 & 1,00 & & 1,00 & \\
\hline $\operatorname{Sim}$ & 27,87 & $0,57(0,43-0,76)$ & & $0,63(0,47-0,84)$ & \\
\hline \multicolumn{2}{|l|}{ Autopercepção da saúde (3) } & & $<0,001 \star \star \star$ & & $<0,001 * \star$ \\
\hline Excelente & 25,81 & 1,00 & & 1,00 & \\
\hline Muito boa & 30,14 & $1,17(0,67-2,02)$ & & $1,01(0,60-1,72)$ & \\
\hline Boa & 40,24 & $1,56(1,01-2,40)$ & & $1,40(0,94-2,10)$ & \\
\hline Regular & 48,18 & $1,87(1,22-2,86)$ & & $1,63(1,09-2,43)$ & \\
\hline Ruim & 65,14 & $2,52(1,64-3,88)$ & & $2,25(1,51-3,36)$ & \\
\hline \multicolumn{2}{|l|}{ Identificação da atividade física como } & & 0,52 & & 0,51 \\
\hline \multicolumn{6}{|l|}{ benefício para saúde (3) \# } \\
\hline Não & 47,10 & 1,00 & & 1,00 & \\
\hline Sim & 45,40 & $0,96(0,86-1,08)$ & & $0,96(0,86-1,08)$ & \\
\hline \multicolumn{2}{|c|}{ Prescrição de atividade física no último ano (4) } & & 0,006 & & 0,04 \\
\hline Não & 48,88 & 1,00 & & 1,00 & \\
\hline Sim & 40,85 & $0,84(0,73-0,95)$ & & $0,88(0,77-0,99)$ & \\
\hline
\end{tabular}

* Corresponde ao nível da variável no modelo hierárquico - cada associação foi ajustada para as variáveis do mesmo nível ou de nível superior no modelo;

** Teste de tendência;

*** Para idosos, estão agrupadas as categorias da variável escolaridade: fundamental completo e ensino médio incompleto, assim como médio completo e superior;

\# Mencionou a atividade física como um dos três fatores mais importantes para a manutenção da saúde.

crição para prática de atividade física no último ano apresentaram proteção para sedentarismo em comparação aos que não receberam tal prescrição.

Quanto ao modelo de atenção, o percentual médio de sedentarismo em adultos residentes nas áreas de abrangência de unidades básicas de saúde do modelo tradicional foi de $30,3 \%$; nas unidades básicas de saúde de PSF o percentual foi de $34,7 \%$ pré-PROESF e 29,9\% pós-PROESF $(\mathrm{p}=0,01)$. Entre os idosos, as respectivas proporções foram 54,4\%, 63,8\% e 54,9\% (p < 0,001).

Nas áreas onde o percentual médio de sedentarismo dos profissionais de saúde foi $0 \%, 26,9 \%$ dos adultos foram classificados como sedentários. Nas unidades onde o percentual médio de sedentarismo nos profissionais de saúde esteve entre $90,1 \%$ e $100 \%$, a prevalência de sedentarismo em 
Prevalência de sedentarismo, análise bruta e ajustada para adultos e idosos na população da área de abrangência de unidades básicas de saúde de municípios da Região Nordeste do Brasil, 2005.

\begin{tabular}{|c|c|c|c|c|c|}
\hline \multirow[t]{2}{*}{ Variáveis (nível) * } & \multirow[b]{2}{*}{$\%$} & \multicolumn{2}{|c|}{ Análise bruta } & \multicolumn{2}{|c|}{ Análise ajustada } \\
\hline & & RP (IC95\%) & $p$ & RP (IC95\%) & $p$ \\
\hline \multicolumn{6}{|l|}{ Adultos } \\
\hline Sexo (1) & & & $<0,001$ & & $<0,001$ \\
\hline Masculino & 43,63 & 1,00 & & 1,00 & \\
\hline Feminino & 34,86 & $0,80(0,72-0,89)$ & & $0,80(0,72-0,89)$ & \\
\hline Idade (anos) (1) & & & $<0,001$ ** & & $<0,001$ ** \\
\hline $30-40$ & 32,27 & 1,00 & & 1,00 & \\
\hline $41-50$ & 36,25 & $1,03(0,90-1,18)$ & & $1,02(0,90-1,18)$ & \\
\hline $51-64$ & 46,50 & $1,32(1,16-1,49)$ & & $1,31(1,16-1,48)$ & \\
\hline \multicolumn{6}{|l|}{$65-70$} \\
\hline \multicolumn{6}{|l|}{$71-75$} \\
\hline \multicolumn{6}{|l|}{$76-79$} \\
\hline \multicolumn{6}{|l|}{80 ou +} \\
\hline Cor de pele (1) & & & 0,001 & & 0,002 \\
\hline Branca & 42,34 & 1,00 & & 1,00 & \\
\hline Não branca & 35,31 & $0,83(0,75-0,93)$ & & $0,84(0,75-0,94)$ & \\
\hline Escolaridade (1) ${ }^{\star \star \star}$ & & & $0,20 * \star$ & & 0,34 ** \\
\hline Não estudou & 41,32 & 1,00 & & 1,00 & \\
\hline Fundamental incompleto & 38,98 & $0,94(0,88-1,08)$ & & $0,94(0,82-1,07)$ & \\
\hline Fundamental completo & 39,15 & $0,95(0,77-1,16)$ & & $0,96(0,78-1,19)$ & \\
\hline Médio incompleto & 37,25 & $0,90(0,69-1,18)$ & & $0,95(0,73-1,25)$ & \\
\hline Médio completo & 35,26 & $085(0,71-1,02)$ & & $0,87(0,72-1,05)$ & \\
\hline Superior & 41,46 & $1,00(0,76-1,32)$ & & $0,99(0,75-1,32)$ & \\
\hline Tabagismo (1) & & & 0,66 & & 0,58 \\
\hline Não, nunca fumou & 38,10 & 1,00 & & 1,00 & \\
\hline Já fumou & 40,38 & $1,06(0,93-1,20)$ & & $0,96(0,84-1,10)$ & \\
\hline Fumante atual & 39,13 & $1,03(0,90-1,18)$ & & $0,96(0,84-1,11)$ & \\
\hline Padrão de consumo sócio-econômi & & & 0,22 ** & & 0,24 \\
\hline A, B ou C & 40,71 & 1,00 & & 1,00 & \\
\hline $\mathrm{D}$ & 38,39 & $0,94(0,82-1,09)$ & & $0,93(0,80-1,09)$ & \\
\hline$E$ & 37,44 & $0,92(0,80-1,05)$ & & $0,91(0,77-1,07)$ & \\
\hline Situação conjugal (1) & & & 0,79 & & 0,25 \\
\hline Casado(a)/Com companheiro(a) & 38,88 & 1,00 & & 1,00 & \\
\hline Viúvo(a) & 39,85 & $1,02(0,80-1,27)$ & & $0,98(0,77-1,24)$ & \\
\hline Separado(a)/Divorciado(a) & 36,54 & $0,94(0,78-1,14)$ & & $0,99(0,81-1,20)$ & \\
\hline Solteiro(a) & 41,2 & $1,06(0,90-1,25)$ & & $1,14(0,96-1,35)$ & \\
\hline Renda familiar per capita (salário mí & & & 0,53 ** & & 0,98 ** \\
\hline Sem renda & 47,92 & 1,00 & & 1,00 & \\
\hline Até 1 & 37,21 & $078(0,66-0,91)$ & & $0,78(0,66-0,92)$ & \\
\hline $1,01-2$ & 38,92 & $0,83(0,67-1,03)$ & & $0,78(0,61-0,98)$ & \\
\hline $2,01-3$ & 40,82 & $0,85(0,59-1,23)$ & & $0,81(0,55-1,18)$ & \\
\hline $3 \mathrm{ou}+$ & 57,14 & $1,19(0,88-1,61)$ & & $1,15(0,83-1,60)$ & \\
\hline Trabalho remunerado no último mê & & & 0,06 & & 0,003 \\
\hline Não, licença ou nunca trabalhou & 40,92 & 1,00 & & 1,00 & \\
\hline Sim & 36,84 & $0,90(0,81-1,00)$ & & $0,84(0,75-0,94)$ & \\
\hline Autopercepção da saúde (3) & & & $0,002 * \star$ & & $0,004 * \star$ \\
\hline Excelente & 35,23 & 1,00 & & 1,00 & \\
\hline Muito boa & 34,02 & $0,97(0,67-1,39)$ & & $1,03(0,71-1,49)$ & \\
\hline Boa & 35,83 & $1,02(0,78-1,32)$ & & $1,06(0,81-1,38)$ & \\
\hline Regular & 39,91 & $1,13(0,88-1,46)$ & & $1,17(0,90-1,52)$ & \\
\hline Ruim & 47,69 & $1,35(1,08-1,78)$ & & $1,38(1,04-1,83)$ & \\
\hline
\end{tabular}

(continua) 


\begin{tabular}{|c|c|c|c|c|c|}
\hline \multirow[t]{2}{*}{ Variáveis (nível) * } & \multirow[b]{2}{*}{$\%$} & \multicolumn{2}{|c|}{ Análise bruta } & \multicolumn{2}{|c|}{ Análise ajustada } \\
\hline & & RP (IC95\%) & $p$ & RP (IC95\%) & $p$ \\
\hline \multicolumn{6}{|l|}{ Identificação da atividade física como } \\
\hline benefício para saúde (3) \# & & & 0,03 & & 0,01 \\
\hline Não & 34,80 & 1,00 & & 1,00 & \\
\hline Sim & 40,20 & $1,16(1,01-1,32)$ & & $1,18(1,04-1,34)$ & \\
\hline \multicolumn{2}{|c|}{ Prescrição de atividade física no último ano (4) } & & 0,65 & & 0,32 \\
\hline Não & 38,37 & 1,00 & & 1,00 & \\
\hline Sim & 39,56 & $1,03(0,90-1,17)$ & & $0,92(0,77-1,09)$ & \\
\hline \multicolumn{6}{|l|}{ Idosos } \\
\hline Sexo (1) & & & 0,73 & & 0,29 \\
\hline Masculino & 67,92 & 1,00 & & 1,00 & \\
\hline Feminino & 67,20 & $0,99(0,93-1,05)$ & & $0,97(0,90-1,03)$ & \\
\hline Idade (anos) (1) & & & $<0,001$ ** & & $<0,001 * *$ \\
\hline \multicolumn{6}{|l|}{$30-40$} \\
\hline \multicolumn{6}{|l|}{$41-50$} \\
\hline \multicolumn{6}{|l|}{$51-64$} \\
\hline $65-70$ & 56,70 & 1,00 & & 1,00 & \\
\hline $71-75$ & 66,03 & $1,16(1,07-1,27)$ & & $1,16(1,06-1,26)$ & \\
\hline $76-79$ & 74,48 & $1,31(1,21-1,43)$ & & $1,30(1,20-1,42)$ & \\
\hline 80 ou + & 84,01 & $1,48(1,37-1,60)$ & & $1,46(1,35-1,58)$ & \\
\hline Cor de pele (1) & & & 0,89 & & 0,78 \\
\hline Branca & 67,58 & 1,00 & & 1,00 & \\
\hline Não branca & 67,29 & $1,00(0,94-1,06)$ & & $1,01(0,95-1,08)$ & \\
\hline Escolaridade (1) $* \star \star$ & & & 0,004 & & 0,15 \\
\hline Não estudou & 70,05 & 1,00 & & 1,00 & \\
\hline Fundamental incompleto & 63,15 & $0,90(0,84-0,97)$ & & $0,93(0,87-1,00)$ & \\
\hline \multicolumn{6}{|l|}{ Fundamental completo } \\
\hline Médio incompleto & 61,19 & $0,87(0,72-1,06)$ & & $0,96(0,79-1,17)$ & \\
\hline \multicolumn{6}{|l|}{ Médio completo } \\
\hline Superior & 61,43 & $0,88(0,73-1,06)$ & & $0,96(0,79-1,16)$ & \\
\hline Tabagismo (1) & & & 0,08 & & 0,6 \\
\hline Não, nunca fumou & 66,42 & 1,00 & & 1,00 & \\
\hline Já fumou & 69,85 & $1,05(0,99-1,12)$ & & $1,12(0,95-1,09)$ & \\
\hline Fumante atual & 63,55 & $0,96(0,87-1,05)$ & & $0,96(0,87-1,16)$ & \\
\hline \multicolumn{2}{|c|}{ Padrão de consumo sócio-econômico (1) } & & 0,8 & & 0,23 \\
\hline A, B ou C & 69,89 & 1,00 & & 1,00 & \\
\hline $\mathrm{D}$ & 66,20 & $0,95(0,86-1,04)$ & & $0,93(0,84-1,03)$ & \\
\hline E & 67,93 & $0,97(0,89-1,66)$ & & $0,93(0,85-1,02)$ & \\
\hline \multicolumn{2}{|l|}{ Situação conjugal (1) } & & $<0,001$ & & 0,22 \\
\hline Casado(a)/Com companheiro(a) & 63,27 & 1,00 & & 1,00 & \\
\hline Viúvo(a) & 72,34 & $1,15(1,07-1,22)$ & & $1,07(1,00-1,14)$ & \\
\hline Separado(a)/Divorciado(a) & 63,19 & $1,00(0,88-1,13)$ & & $1,01(0,89-1,15)$ & \\
\hline Solteiro(a) & 67,57 & $1,07(0,93-1,23)$ & & $1,06(0,93-1,22)$ & \\
\hline \multicolumn{2}{|c|}{ Renda familiar per capita (salário mínimo) (1) } & & 0,74 ** & & 0,91 ** \\
\hline Sem renda & 68,97 & 1,00 & & 1,00 & \\
\hline Até 1 & 67,33 & $0,98(0,84-1,13)$ & & $0,94(0,81-1,09)$ & \\
\hline $1,01-2$ & 68,88 & $1,00(0,84-1,18)$ & & $0,97(0,81-1,16)$ & \\
\hline $2,01-3$ & 58,97 & $0,85(0,64-1,15)$ & & $0,86(0,63-1,18)$ & \\
\hline 3 ou + & 77,42 & $1,12(0,89-1,42)$ & & $1,04(0,80-1,34)$ & \\
\hline Trabalho remunerado no último mê & & & 0,06 & & 0,38 \\
\hline Não, licença ou nunca trabalhou & 67,91 & 1,00 & & 1,00 & \\
\hline $\operatorname{Sim}$ & 56,98 & $0,84(0,70-1,01)$ & & $0,92(0,76-1,11)$ & \\
\hline
\end{tabular}

(continua) 
entre autopercepção de saúde e nível de atividade física está de acordo com a literatura, indicando que pessoas mais ativas classificam sua saúde de forma mais positiva do que os sedentários ?

Uma das intenções gerais do PROESF é discutir sobre os desfechos em saúde levando em conta o modelo de atenção básica, que se mostrou relevante, uma vez que os moradores em áreas de abrangência de PSF Pré-PROESF eram os mais sedentários. No entanto, a falta de associação entre sedentarismo dos profissionais e sedentarismo de adultos e idosos sugere que o contexto de maior pobreza da população de áreas de abrangência do PSF pode ser uma explicação para o achado ${ }^{3}$. Embora não se descarte o efeito de outras características dos modelos de atenção, essa possibilidade é reforçada pela associação da baixa renda familiar com sedentarismo encontrada neste estudo para adultos e idosos.

Algumas limitações do presente estudo devem ser consideradas. Inicialmente, deve-se ter cuidado em extrapolar nossos achados para a população em geral, visto que nossa amostra é composta por indivíduos residentes em áreas de abrangência de unidades básicas de saúde, o que implica, de acordo com a realidade sanitária brasileira, uma amostra mais pobre do que a população em geral ${ }^{3}$. Nossa taxa de não-resposta inclui não só as pessoas selecionadas nos domicílios que se recusaram a participar do estudo, como também domicílios nos quais não foram encontrados moradores, o que pode gerar uma subestimativa do percentual de não-respondentes. Optamos por analisar os dados de adultos e idosos separadamente exatamente porque a probabilidade de seleção foi diferente para cada grupo; um mesmo número de sujeitos era necessário pelo cálculo de tamanho de amostra em cada faixa etária, embora existam mais adultos do que idosos na população.

Entre as vantagens do presente estudo, podese destacar a abrangência regional da investigação, que incluiu 41 cidades de porte médio ou grande (+100 mil habitantes) em duas regiões do Brasil. Com exceção do inquérito do INCA e da Pesquisa de Padrão de Vida (PPV), não existem estudos com essa abrangência no Brasil 18,21. Outro aspecto positivo é a utilização do IPAQ, um questionário que foi desenvolvido por diversos pesquisadores de vários países (inclusive o Brasil), para produzir dados comparáveis relacionados à prática de atividade física ${ }^{14}$. A opção de utilizarmos um ponto de corte similar ao empregado em outros estudos e de acordo com as recomendações atuais quanto à prática de atividade física aumenta a comparabilidade de nossos dados com estudos prévios e futuros. Outro aspecto fundamental é que o poder estatístico, em geral, foi suficiente para as análises estratificadas por região e grupo etário, permitindo que diferenças relevantes fossem detectadas como estatisticamente significativas.

Um dos projetos atuais do Ministério da Saúde é inserir a prática de atividade física no cotidiano da rede básica de saúde. Algumas iniciativas nacionais, como a Academia da Cidade no Recife, indicam que tal inserção poderia ser útil para a diminuição do sedentarismo na população 23. Finalmente, conclui-se que os níveis de sedentarismo entre os indivíduos residentes em áreas de abrangência de unidades básicas de saúde nos estados do Sul e Nordeste do Brasil são elevados.

Embora as pessoas saibam que a atividade física é importante para a saúde, a prescrição de atividade física por parte de profissionais de saúde dessas unidades ainda é insuficiente para promover alterações na atitude das pessoas em relação à prática de atividade física. Pode-se concluir ainda que as unidades básicas de saúde não estão atendendo as necessidades dos usuários em relação à prática da atividade física como instrumento de promoção da saúde.

Essas observações sugerem que são necessários projetos e ações estruturadas e participativas entre os profissionais e população, além de investimentos por parte da atenção básica brasileira, com objetivo de qualificar e reorganizar o sistema, para melhor atender a população, promovendo a saúde desta no que se refere à prática de atividade física e a conseqüente diminuição do sedentarismo. 


\section{Resumo}

As mudanças na pirâmide demográfica brasileira e conseqüente envelhecimento populacional têm promovido aumento de doenças crônicas. Nesta realidade, as unidades básicas de saúde e a atividade física ganham importância. Em um estudo com delineamento transversal, avaliou-se a prevalência de sedentarismo e fatores associados em 4.060 adultos e 4.003 idosos, residentes em áreas de unidades básicas de saúde de 41 municípios com mais de 100 mil habitantes, em sete estados do Brasil. A prevalência de sedentarismo foi de 31,8\% (IC95\%: 30,4-33,2) e 58\% (IC95\%: 56,4-59,5) para adultos e idosos respectivamente, sendo sempre maior na Região Nordeste e nos homens. Baixa renda familiar foi fator de risco para sedentarismo nos adultos e idosos, enquanto a baixa escolaridade apresentou um efeito somente entre os idosos. Houve relação inversa entre autopercepção de saúde e sedentarismo para adultos e idosos. A prevalência média de sedentarismo foi maior na área de abrangência das unidades básicas de saúde Pré-PROESF em comparação aos outros modelos de atenção básica. Conclui-se que a prevalência de sedentarismo é muito elevada e que os grupos sócio-econômicos mais desfavorecidos apresentam nível menor de atividade física.

Atividade Motora; Centros de Saúde; Adulto; Idoso

\section{Colaboradores}

F. V. Siqueira contribuiu na concepção do artigo, análise de dados, interpretação e redação final do artigo; coordenou o trabalho de campo do estudo e participou da elaboração dos instrumentos e processamento de dados. L. A. Facchini colaborou na revisão do manuscrito. R. X. Piccini, E. Tomasi, E. Thumé e D. S. Silveira participaram da elaboração dos instrumentos e demais etapas do estudo, bem como da revisão do artigo. P. C. Hallal revisou o trabalho, desde sua concepção até a redação final, principalmente na parte específica relacionada à atividade física.

\section{Agradecimentos}

Ao Ministério da Saúde do Brasil e ao Banco Mundial, pelo apoio financeiro.

\section{Referências}

1. Monteiro CA. Novos e velhos males da saúde no Brasil. A evolução do país e de suas doenças. São Paulo: Editora Hucitec/Universidade de São Paulo; 1995.

2. Murray CJ, Lopez AD. Mortality by cause for eight regions of the world: Global Burden of Disease Study. Lancet 1997; 349:1269-76.

3. Facchini LA, Piccini RX, Tomasi E, Thumé E, Silveira DS, Siqueira FV, et al. Desempenho do PSF no Sul e no Nordeste do Brasil: avaliação institucional e epidemiológica da Atenção Básica à Saúde. Ciênc Saúde Coletiva 2006; 11:669-81.

4. Shepard RJ. Physical activity, fitness and health: the current consensus. Quest 1995; 47:228-303.

5. Le Marchand L, Kolonel LN, Yoshizawa CN. Lifetime occupational physical activity and prostate cancer risk. Am J Epidemiol 1991; 133:103-11.

6. Lee IM, Paffenbarger RS, Hsieh Jr. CC. Physical activity and risk of prostatic cancer among college alumni. Am J Epidemiol 1992; 135:169-79.
7. Hallal PC, Victora CG, Wells JC, Lima RC. Physical inactivity: prevalence and associated variables in Brazilian adults. Med Sci Sports Exerc 2003; 35:1894-900.

8. Barros MV, Nahas MV. Comportamentos de risco, auto-avaliação do nível de saúde e percepção de estresse entre trabalhadores da indústria. Rev Saúde Pública 2001; 35:554-63.

9. Garrett NA, Brasure M, Schmitz KH, Schultz MM, Huber MR. Physical inactivity: direct cost to a health plan. Am J Prev Med 2004; 27:304-9.

10. Fuscaldo JM. Prescribing physical activity in primary care. WV Med J 2002; 98:250-3.

11. Chakravarthy MV, Joyner MJ, Booth FW. An obligation for primary care physicians to prescribe physical activity to sedentary patients to reduce the risk of chronic health conditions. Mayo Clin Proc 2002; 77:165-73. 
12. Ministério da Saúde. Termo de referência para o estudo de linha de base nos municípios selecionados para o componente 1 do PROESF. Brasília: Ministério da Saúde; 2004.

13. Craig CL, Marshall AL, Sjostrom M, Bauman AE, Booth ML, Ainsworth BE, et al. International physical activity questionnaire: 12-country reliability and validity. Med Sci Sports Exerc 2003; 35:138195.

14. U.S. Department of Health and Human Services. Physical activity and health: a report of the surgeon general. Atlanta: U.S. Department of Health and Human Services, Centers for Disease Control and Prevention, National Center for Chronic Disease Prevention and Health Promotion; 1996.

15. Barros AJ, Hirakata VN. Alternatives for logistic regression in cross-sectional studies: an empirical comparison of models that directly estimate the prevalence ratio. BMC Med Res Methodol 2003; 3:21.

16. Victora CG, Huttly SR, Fuches SC, Olinto MT. The role of conceptual frameworks in epidemiological analysis: a hierarquical approachI. Int J Epidemiol 1997; 26:224-7.

17. Matsudo SM, Matsudo VR. Nível de atividade física da população do Estado de São Paulo: análise de acordo com o gênero, idade, nível sócio-econômico, distribuição geográfica e de conhecimento. Rev Bras Ciênc Mov 2002;10:41-50.
18. Coordenação de Prevenção e Vigilância, Instituto Nacional de Câncer. Inquérito domiciliar sobre comportamentos de risco e morbidade referida de doenças e agravos não transmissíveis. Brasil, 15 capitais e Distrito Federal 2002-2003. Rio de Janeiro: Instituto Nacional de Câncer; 2004.

19. Barros MV, Nahas MV. Health risk behaviors, health status self-assessment and stress perception among industrial workers. Rev Saúde Pública 2001; 35:554-63.

20. Castanho VS, Oliveira LS, Pinheiro HP, Oliveira HC, de Faria EC. Sex differences in risk factors for coronary hearh disease: a study in a Brazilian population. BMC Public Health 2001; 1:3.

21. Monteiro CA, Conde WL, Matsudo SM, Matsudo VR, Bonsenor IM, Lotufo PA. A descriptive epidemiology of leisure-time physical activity in Brazil, 1996-1997. Rev Panam Salud Pública 2003; 14:24654.

22. Bacchieri G, Gigante DP, Assunção MC. Determinants and patterns of bicycle use and traffic accidents among bicycling workers in Pelotas, Rio Grande do Sul, Brazil. Cad Saúde Pública 2005; 21:1499-508.

23. Secretaria da Saúde, Prefeitura Municipal de Recife. Academia da Cidade. http://www.recife.pe.gov. $\mathrm{br} / \mathrm{pr} / \mathrm{secsaude} /$ academia.php (acessado em 25/ Jan/2007).

Recebido em 13/Fev/2007

Versão final reapresentada em 09/Mai/2007

Aprovado em 16/Mai/2007 